\title{
Bilateral Metastatic Acute Lymphoblastic Leukaemia Presenting as Hypopyon: A Case Report
}

\section{Asmita Indurkar, Manoj Soman, Sameer Iqbal*, Sumita Phatak and Unnikrishnan Nair R}

Department of Vitreo-Retinal Service, Chaithanya Eye Hospital and Research

Institute, Thiruvananthapuram, Kerala, India

*Corresponding Author: Sameer Iqbal, Department of Vitreo-Retinal Service, Chaithanya Eye Hospital and Research Institute, Thiruvananthapuram, Kerala, India.
Received: December 09, 2021

Published: January 31, 2022

(C) All rights are reserved by Sameer Iqbal., et al.

\begin{abstract}
Here we report a case of a 32-year-old male patient with bilateral haemorrhagic hypopyon as an unusual presenting feature of acute lymphoblastic leukaemia (ALL). Cytology of anterior chamber tap was suggestive of leukemic infiltration of the anterior chamber of the eyes, a sign of metastatic acute lymphoblastic leukemia. He was conservatively managed with topical steroids and cycloplegics. Patient was already initiated with chemotherapy under the care of an oncologist. A leukemic hypopyon is considered as an early sign of central nervous system involvement or systemic relapse. A finding of clinical significance, it should be immediately reported to the oncologist to urgently rule out CNS involvement and for aggressive management.
\end{abstract}

Keywords: Acute Lymphoblastic Leukaemia; Hypopyon; Acute Myeloblastic Leukemia

\section{Introduction}

Orbital and ocular lesions are the third most frequent extramedullary locations of acute leukemia after the meninges and testicles [1]. Ocular manifestations may occur from thrombo-embolic phenomena due to a hyper-viscosity state (eg: retinal artery or retinal vein occlusion) or from direct invasion of the malignant cells to ocular tissue [2]. Hypopyon uveitis can be an indication of relapsing leukemia and occurs in association with other ocular or systemic manifestations [3]. Bilateral hypopyon as a presenting feature in chronic myeloid leukemia (CML) [3], in relapses of CML [2], acute myeloblastic leukemia (AML) [4] and acute lymphoblastic leukemia (ALL) [5] has been reported however it has never been reported as a presenting feature of ALL. We report a case of a 32-year-old male who presented to us with bilateral haemorrhagic hypopyon with cytological confirmation of ALL.

\section{Case Report}

A 32-year-old male was referred to us with complaints of insidious onset diminution of vision and redness in both eyes for
15 days. He gave a history of recent episode of fever 5 days back and was diagnosed as acute lymphoblastic leukemia (ALL) 20 days back. He had undergone two infusions of chemotherapy since the diagnosis, details of which were unknown due to lack of records with the patient.

On presentation his corrected distance visual acuity was counting finger close to face in the right eye and 20/80 in the left eye. Intra-ocular pressure on applanation tonometry was $28 \mathrm{~mm}$ hg in both eyes. Anterior segment (Figure 1) was significant for conjunctival congestion with central corneal epithelial edema in both eyes. A haemorrhagic hypopyon measuring $1 \mathrm{~mm}$ was present in both eyes. Additionally, a white dense coagulum was present in both eyes. It covered the visual axis in the right eye, and was present along the inferior pupillary margin of the left eye. On dilated fundus examination there was no view in the right eye and only superior retinal quadrants were visible in the left eye which appeared normal. An ultrasound B scan was done in both eyes which demonstrated an attached retina with no retino-choroidal thickening or vitreous echoes. 


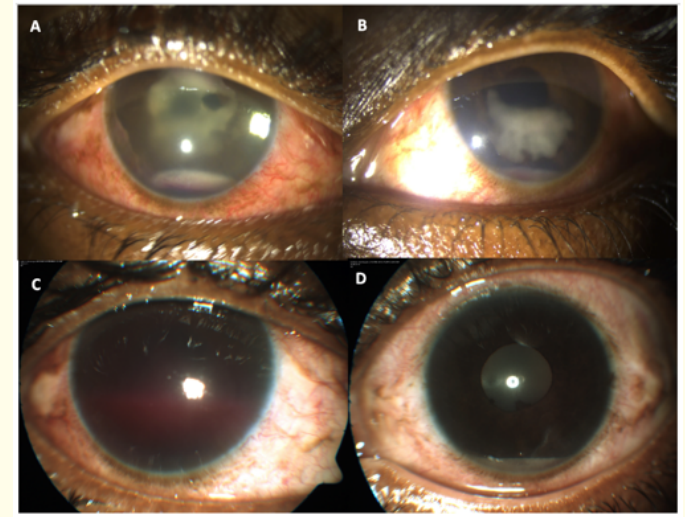

Figure 1: A (Right eye), B (Left eye) - Anterior segment examination at presentation showing haemorrhagic hypopyon in both eyes and white dense coagulum covering the visual axis in the right eye and along the inferior papillary margin in the left eye. $\mathrm{C}$ (Right eye), D (Left eye): Follow up images showing evolvement of haemorrhagic hypopyon to hyphaema in the right eye and resolved pupillary exudates in the left eye.

We decided to obtain an aqueous specimen to define the nature of the cellular infiltrate and to rule out endogenous infection. Bilateral AC tap was therefore sent for microbiology (Gram stain, $\mathrm{KOH}$ wet mount, bacterial and fungal culture, and sensitivity) and cytopathology. Patient was started on topical antibiotics and cycloplegics in the both eyes. We deferred anti-glaucoma medications (AGM) as we had performed an anterior chamber paracentesis. He was already on systemic broad-spectrum antibiotics which were advised to continue.

He presented the next day for a follow-up. Repeat B-scan showed the development of localised retino-choroidal elevations in the right eye, while the left eye remained the same. At this point we had a differential diagnosis of metastatic leukemia or an endogenous endophthalmitis in a patient who recently underwent chemotherapy and was under systemic broad spectrum antibiotic cover. He was maintained on topical steroid eye drops, cycloplegics and topical antibiotics and advised to continue systemic antibiotics. Two days later, while waiting for the anterior chamber tap reports, the haemorrhagic hypopyon evolved into a hyphaema in the right eye while the pupillary exudates completely disappeared in the left eye (Figure $1 \mathrm{C}$ and D). Persistently high intraocular pressure (26 $\mathrm{mm} \mathrm{hg)} \mathrm{was} \mathrm{noted} \mathrm{in} \mathrm{the} \mathrm{right} \mathrm{eye} \mathrm{for} \mathrm{which} \mathrm{topical} \mathrm{antiglaucoma}$ medications were started. On dilated fundus examination there was still no view in the right eye; a new solitary haemorrhage was noted in the left eye supero-temporal peripheral fundus. B-scan of the right eye (Figure 2) showed persistent retino-choroidal elevations with development of overlying subretinal fluid pockets. Meanwhile, the laboratory reports confirmed that culture yields were negative for bacterial and fungal growth. Cytology showed cells with cleaved nuclei and high nuclear/cytoplasmic ratio, scant cytoplasm and few cells with prominent nucleoli (Figure 3) which was suggestive of leukemic infiltration of the anterior chamber of the eyes, a sign of metastatic acute lymphoblastic leukemia.

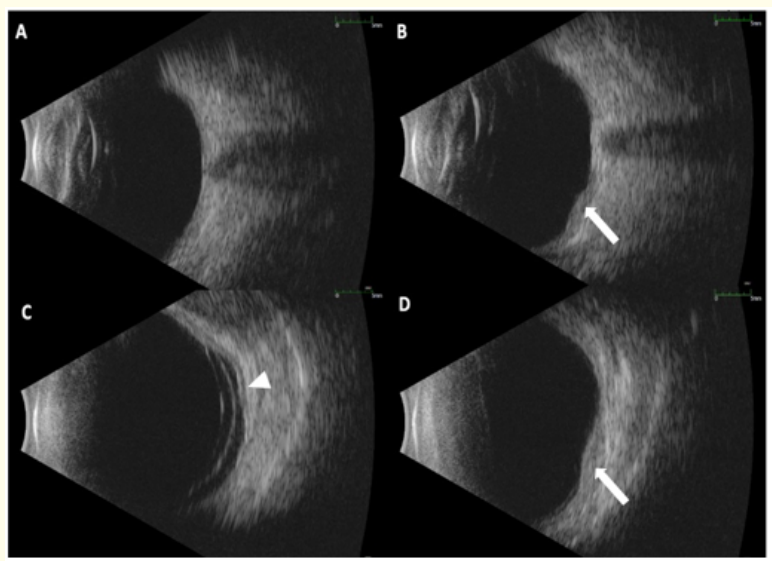

Figure 2: A; B-scan of right eye at presentation was normal. B,C,D; Follow up B-scan of right eye showing localised retino-choroidal elevations (arrows) with overlying subretinal fluid pockets (arrow head).

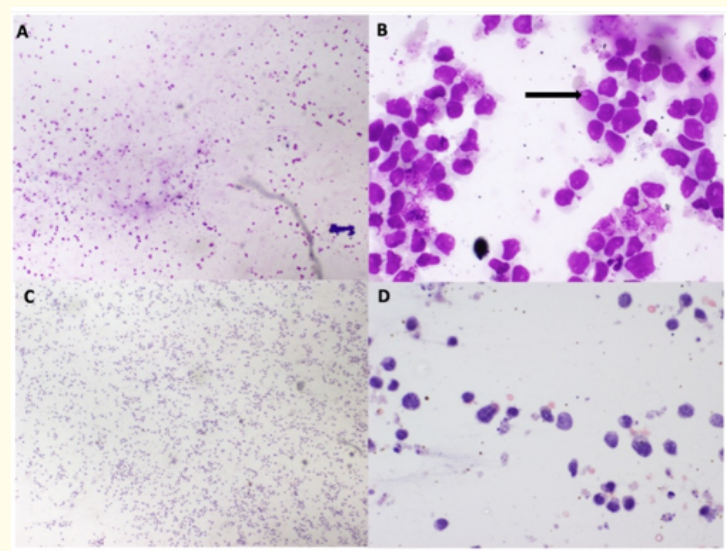

Figure 3: A - Giemsa: 100x Cellular smear showing lymphoid cell population. B - Giemsa 400x: Cells showing cleaved nuclei with high nucleo-cytoplasmic (N/C) ratio, scant cytoplasm and few with prominent nucleoli (arrow). C - Pap 100x: Dense cellular infiltrate with scant background stroma. D - Pap 400x: Cells with high N/C ratio with interspersed inflammatory cells. 
Based on this report the oncologist was requested to urgently rule out CNS involvement while conservative management of hyphaema was continued. In the meantime, within the next couple of days, the patient's systemic status deteriorated rapidly, and the patient expired.

\section{Discussion and Conclusion}

Our case is unique in a way that a bilateral hemorrhagic hypopyon was seen at primary presentation in acute lymphoblastic leukemia and soon after which the systemic condition of the patient deteriorated significantly and rapidly. The diagnosis of ALL was complete with histopathological documentation. Ocular involvement in Leukemia has been seen to be associated with CNS involvement in relapsed ALL. This case seemed to be an aggressive primary presentation of ALL with bilateral simultaneous blood tinged hypopyon with systemic deterioration of the patient.

Usually, systemic manifestations and diagnosis is evident before the ocular signs of leukemia [3]. Hemorrhagic or blood tinged hypopyon, absence of posterior synechiae and raised intro-ocular pressure are features of hypopyon in metastatic or masquerade syndrome [6], all which were present in our patient. Our patient also had a clear vitreous cavity and retino-choroidal thickening which is also typical of masquerade syndromes and helps to differentiate it from endogenous endophthalmitis [3].

Ocular involvement of the iris and anterior chamber as the site of primary disease is a rare event. Despite the rarity, presentation of a blood tinged hypopyon should instantly be investigated to rule out leukemia as one of the significant differentials. The anterior chamber serves as a sanctuary for cancer cells, and treatment protocols, including better anterior chamber prophylaxis, are warranted [7]. A corticosteroid-resistant hypopyon, even if unilateral, should prompt an anterior chamber tap for both cytology and culture [5].

\section{Bibliography}

1. Charif Chefchaouni M., et al. "Ophthalmic manifestations of acute leukemia”. Journal Français D'Ophtalmologie 25 (2002): 62-66.

2. Ben-Simon GJ and Barequet IS. "Anterior uveitis as presenting sign of myeloproliferative disease". Annals of Ophthalmology 35 (2003): 62-64.
3. Sudharshan S., et al. "Bilateral Hypopyon as the Presenting Feature of Chronic Myeloid Leukemia". Ocular Immunology and Inflammation 16 (2008): 244-246.

4. Foster CS., et al. "Relapsing Acute Myeloid Leukemia Manifesting as Hypopyon Uveitis". American Journal of Ophthalmology 119 (1995): 361-364.

5. Yi DH., et al. "Acute unilateral leukemic hypopyon in an adult with relapsing acute lymphoblastic leukemia". American Journal of Ophthalmology 139 (2005): 719-721.

6. Ramsay A and Lightman S. "Hypopyon uveitis". Survey of Ophthalmology 46 (2001): 1-18.

7. Decker EB and Burnstine RA. "Leukemic relapse presenting as acute unilateral hypopyon in acute lymphocytic leukemia”. Annals of Ophthalmology 25 (1993): 346-349.

\section{Assets from publication with us}

- Prompt Acknowledgement after receiving the article

- Thorough Double blinded peer review

- Rapid Publication

- Issue of Publication Certificate

- High visibility of your Published work

Website: www.actascientific.com/

Submit Article: www.actascientific.com/submission.php Email us: editor@actascientific.com

Contact us: +919182824667 